\title{
Une présentation du Languedoc-Roussillon
}

Robert Ferras, Dominique Borne, Pierre Desplanques, Tony Male et Clara Dematteis

\section{(2) OpenEdition}

\section{Journals}

Édition électronique

URL : http://journals.openedition.org/trema/2280

DOI : 10.4000/trema.2280

ISSN : 2107-0997

Éditeur

Faculté d'Éducation de l'université de Montpellier

Édition imprimée

Date de publication : 1 mai 1994

Pagination : 45-76

ISSN : 1167-315X

Référence électronique

Robert Ferras, Dominique Borne, Pierre Desplanques, Tony Male et Clara Dematteis, « Une présentation du Languedoc-Roussillon », Tréma [En ligne], 5 | 1994, mis en ligne le 01 mai 1994, consulté le 19 avril 2019. URL : http://journals.openedition.org/trema/2280 ; DOI : 10.4000/ trema.2280

Ce document a été généré automatiquement le 19 avril 2019. 


\title{
Une présentation du Languedoc- Roussillon
}

\author{
Robert Ferras, Dominique Borne, Pierre Desplanques, Tony Male et Clara \\ Dematteis
}

\section{Robert Ferras}

1 Le recours aux textes fondateurs, ceux que l'on cite périodiquement sans trop aller y voir de plus près, est riche d'enseignements dans l'approche d'une région, et pour deux simples raisons. Ils permettent de voir ce qui se répète, à l'infini, et de déceler ce qui innove, dans le vrai comme dans le faux. La démarche n'implique aucun passéisme, elle traduit seulement l'envie de replacer les choses en situation, dans leur temps, dans l'air de leur temps.

\section{Pourquoi, comment, une archéologie du savoir?}

2 1. De tout temps et très tôt, par grandes catégories, écrivains, voyageurs, grands administrateurs ont donné périodiquement un point de vue que l'on peut considérer aussi comme étant de géographe, parfois sans trop le savoir. Le géographe, au sens moderne du terme, n'interviendra que plus tard, remettant en forme selon ses propres ingrédients, son approche propre, un discours parfois très ancien. Ils éclairent bien les choix actuels. Il y a à glaner dans le Pro Fonteio de Cicéron quand il nous parle de la Narbonnaise, dans les itinéraires d'Arthur Young à la veille de la Révolution ou ceux de R. L. Stevenson un peu plus tard, dans les rapports des intendants de Languedoc comme Basville ou Ballainvilliers, réédités il y a peu.

On en retiendra tout un ensemble d'expressions un peu baroques, redondantes ou en rupture, mais dont il faut bien tenir compte, car même la « langue de bois » n'est jamais gratuite. 
Au plan du cadre physique, considéré longtemps comme point de départ obligatoire :

- l'amphithéâtre, qui charpenterait le Languedoc et les degrés de « l'escalier » menant de la montagne à la mer en passant par la garrigue, haute et basse, jusqu'à la plaine, elle même haute et basse ;

- les moyennes climatiques, qui n'ont ici aucune signification de type «tempéré », le climat méditerranéen se caractérisant (à Nîmes, à Vaison ou ailleurs comme le démontrent en ce moment les journaux) d'abord en termes d'excès et... précisément, d'écarts à la moyenne ;

- la garrigue, pétrée (ce qui évoque depuis les auteurs anciens une Arabie du même nom) et répulsive jusqu'à ce que son image bascule à travers les vieilles routes que reprennent les " périurbains » vers Saint-Martin de Londres ou Saint-Mathieu de Tréviers, plus les routes touristiques vraies ou fausses, des verriers, du vin, quand le canton rural part à la poursuite d'une économie ancienne jusque là superbement ignorée ;

- la montagne, figée, vidée, hiératique, sorte de conservatoire ethnologique où rien ne bougerait depuis que « tout le monde » en est parti, où l'on semble faire fi de toute l'ampleur des mutations foncières enregistrées, sorte de parc sous cellophane, national ou régional.

Au plan des hommes et de leurs activités :

- la ville lieu-de-tous-les-records, Montpellier si proche et si éloignée de Nîmes, Narbonne face à Béziers, Agde la Grecque démultipliée en Cap, en Grau, vêtue ou dénudée, Pézenas petite mais du « Grand Siècle », Lunel tout contre Sommières... Sans parler des villes du piémont qui n'existent plus guère. Le plus bel exemple étant fourni par Lodève, présente partout depuis la plus haute Antiquité en son Pagus lutevensis, et désormais à peu près absente de partout, moins favorisée que Ganges, trop proche de Montpellier, dépassée par Clermont-l'Hérault et dont le rôle d'étape au pied du Larzac ne débouche guère que sur Le Caylar ;

- le chef-lieu de canton, lieu de toutes les surenchères, qui doit absolument -pour cause de survie- garder sa gendarmerie ou tel service. Ce qu'on ne peut lui reprocher. Ou s'inventer telle ou telle spécialité pour paraître, ce qui ne manque pas de surprendre en termes de mystification, de géographismes. Que pèsent Florensac et Bessan en basse vallée de l'Hérault ? Le poids d'un gros bourg, ce qui n'est déjà pas si mal, chacun avec sa spécificité. Doit-on vraiment dire, pour émerger clans un discours que l'on croit volontiers valorisant, comme à Claret, que provient de là le vin du même nom ? Ou, même pire, qu'est-ce que cette « vallée dorée » dont personne n'a jamais entendu parler hors des dépliants qui lui sont consacrés?

6 Tous les carrefours, tous les hauts lieux au nombre sans cesse croissant, parce que de fabrication autochtone, ont généré un sottisier inépuisable. Que l'on songe ainsi à la filière cathare! Au pire, tous les clichés sont bons, que le géographe se doit de cultiver, de réinjecter dans son discours sur l'espace, en raison des « indicateurs idéologiques » qu'ils représentent.

7 Il manquait la wine connection, la voici, avec l'usine à vin et la mer de vigne, que confortent tant de cartes de degré de viticolité ou autres taux. Est appelée au secours, là encore, l'Antiquité et le désir de s'enraciner au plus lointain, quand on rappelle les découvertes d'amphores à Ostie, ayant transporté du vin vieux de Béziers, quand on suppose la vigne autochtone parce que retrouvée dans des tufs anciens proches de Montpellier.

2. Comment prendre en compte cela et le dépasser, tout en l'utilisant ? 
A partir de sources faciles d'accès :

- les textes fondateurs produits par des géographes contemporains. Nous laisserons de côté les autres, plus anciens, et qui méritent recensement et étude fine. Deux auteurs, pour l'essentiel, fondent cette région. Le premier, à petite échelle, incluant l'ensemble du Midi méditerranéen français, le second à grande échelle, à cheval sur deux départements seulement, Hérault et Gard. Soit Jules Sion, dans les années 1930 en forme de petit manuel publié chez Colin, et Raymond Dugrand, dans les années 1960 en forme de thèse publiée aux PUF sur villes et campagnes en Bas-Languedoc. Les autres publications, intermédiaires et rares, ou postérieures et plus nombreuses, doivent être revues, avec profit, à la lumière de ces textes.

- deux atlas qui permettent pour la première fois de connaître en détail le LanguedocRoussillon, l'atlas dirigé par Raymond Dugrand et l'atlas dirigé par Roger Brunet, qui font référence. On l'a oublié, mais jusque-là aucune vision d'ensemble du Languedoc-Roussillon ne se trouvait nulle part. On comprend dès lors le succès de ce type de publication, on comprend moins que leur diffusion ne soit pas à la hauteur d'un contenu tout à fait exceptionnel.

10 Atlas c'est-à-dire dessin donnant à lire cette région, mais dont la cartographie n'est pas l'élément le plus important, s'il reste le plus spectaculaire. La donner à lire mais aussi la connaître et -en jouant sur les mots- à travers ses desseins, lesquels renvoient aux acteurs et à leurs stratégies.

- des thèses. Sur le vignoble et dans le cadre de la géographie seulement (car les économistes et autres spécialistes en ont produit autant) on en compte quatre, en quelques années et selon des éclairages différents. Raymond Dugrand, déjà cité, traite des rapports villecampagne et produit, à partir du dépouillement minutieux de tout ce qui a trait à la propriété foncière et à l'absentéisme viticole, la première thèse moderne sur la région. Gaston Galtier se situe dans une optique monographique et comparative à la fois pour étudier un vignoble de masse face à l'Italie, l'Espagne, l'Argentine, etc. Franck Auriac revoit les choses à la lumière d'une approche systémique. Pierre Carrière, dans le cadre d'une comparaison entre l'agriculture de l'URSS et celle du Languedoc-Roussillon, propose une quatrième version. Les relire en parallèle est riche d'enseignements sur le contexte scientifique qui a présidé à leur production. Songer à l'élevage en Normandie ou sur les hautes terres du Massif central renvoie automatiquement à un spécialiste et un seul, Armand Frémont ou André Fel. Rien de tel ici, où, à l'ombre de Raymond Dugrand, les discours sur la région sont multiples. Pourrait-on trouver un deuxième exemple en France, de région ayant suscité sur le même thème autant de travaux en si peu de temps ? Replacés dans un contexte épistémologique ils ne manquent pas d'intérêt.

- les manuels et « grandes » collections qui se font l'écho de ces démarches méritent relecture attentive sur plusieurs décennies. On songe, d'abord, à l'entreprise d'Ardouin-Dumazet. En tête par la diffusion se placent le « Découvrir la France » dirigé chez Larousse par Roger Brunet (Raymond Dugrand, Robert Ferras), et « l'Atlas et Géographie de la France moderne » dirigé chez Flammarion par Louis Papy (Robert Ferras, Henri Picheral, Bernard Vielzeuf), deux collections menées à leur terme et couvrant l'ensemble du territoire national. Parmi les derniers exemples, aux PUF (Christian Verlaque) chez Bréal (Robert Ferras, Jean-Paul Volle). Alternent dans ce domaine des approches très variées, et chacun s'appliquera à discerner ce qui lui convient le mieux au gré du temps, mais le meilleur manuel n'est-il pas celui que l'on fait pour soi? 
11 3. Le Languedoc-Roussillon, en 1993, c'est finalement, dans le domaine de l'approche géographique, trois entrées possibles relevant :

- de l'institutionnel, avec des limites administratives précises et leurs caractéristiques ; il s'agit de cinq départements plus ou moins bien accolés et eux-mêmes si divers ;

- $\mathrm{du}$ fonctionnel, autour d'un réseau urbain sorti d'une organisation cellulaire ancienne pour se réorganiser sous la direction d'une capitale régionale, Montpellier ; ce qui met l'accent sur un secteur oriental nettement plus dynamique ;

- du « mental » à travers tout le discours tenu, et toutes les représentations qui vont avec.

Il en découle un certain nombre de contradictions entre des territoires normés et une mémoire territoriale qui diffère. C'est là-dessus qu'il faut mettre l'accent, pour ne plus en parler : désormais et pour la première fois la construction du Languedoc-Roussillon est claire, avec des limites administratives -comme pour tout autre région- qui incluent ici quatre départements littoraux, Pyrénées-Orientales, Aude, Hérault et Gard et un département montagnard, la Lozère. Au total plus de 2 millions d'habitants. L'entité Languedoc-Roussillon, quels que soient les jugements que l'on porte sur elle, est là, et fait tomber tout un pan d'états d'âme exprimés par les géographes dans leurs travaux sur ses limites. Faisons avec la région que l'on a. Que retenir du Languedoc-Roussillon ? Deux remarques préalables :

- cette construction n'a jamais été totalement fixée et à l'heure actuelle on peut partir de deux hypothèses parfaitement opposées pour en parler. Le Languedoc-Roussillon n'est qu'un agrégat de circonstance donc artificiel. Ou bien, le Languedoc-Roussllon existe et il nous arrive de le rencontrer.

- la terminologie, double, révèle qu'il y a au moins un Roussillon et un Languedoc. En fait c'est bien plus compliqué, les 3 départements littoraux actuels, Aude, Hérault, Gard, ne sont en fait qu'un héritage d'un Bas-Languedoc face à un ancien Haut-Languedoc dont la capitale était Toulouse.

Se superposent à ces évidences des surenchères médiatiques comme

- le sud intense

- Montpellier la surdouée

- la route du sel

- le vin des cathares

- et tant d'autres, qui ne sont pas sans intérêt, bien au contraire. Les écrivains anciens et les publicistes actuels y vont de leurs hauts lieux à usage touristique, en témoigne cette « île singulière ", citation empruntée à Paul Valéry qui marquait il y a longtemps l'entrée de Sète. De même au temps des vieilles rivalités lisait-on à l'entrée de Béziers, « Béziers, capitale du vin » et à l'entrée de Narbonne, « Narbonne, capitale du vin pur. » Pézenas est ville d'Etats, Narbonne capitale de la Narbonnaise, Nîmes Rome ou Madrid française et Béziers Séville française. Montpellier se contentant d'être surdouée, y compris en matière de marketing urbain.

14 La proposition serait mince et naïve si elle consistait à dire qu'il est difficile de laisser la primauté -systématiquement- à la géographie physique, en fait à la géomorphologie. Cela remet en cause tout le vieux système " géographique » édifié à partir de la vieille coupure plaine (au sens étroit) et altitude ; on en retiendra, et c'est là qu'on voulait en venir, qu'il $\mathrm{y}$ a bien des manières d'entrer en géographie du Languedoc-Roussillon. 
15 Au terme de cette introduction il vaut mieux se poser la question : quels concepts, les plus opératoires possibles, utiliser? C'est peut-être là la vraie possibilité de bâtir une leçon destinée à des étudiants.

Alors que pour des élèves on les explicite, pour des étudiants on les énonce.

On en retiendra 8, par groupes de deux, qui constitueraient l'essentiel d'une démarche géographique actuelle sur la région :

- échelles et champs

- acteurs-stratégies

- flux-diffusion

- paysage-représentations.

18 Ajoutons à ce choix qu'il faut aussi prendre en compte un discours actuel, le discours que l'on peut attendre du géographe. Alors que le Languedoc-Roussillon reste encore une unité composite d'appellations plus ou moins bien contrôlées, ses composantes s'inscrivent dans des champs nouveaux qui parlent d'arc, de pôles, de nœuds et de centres, de carrefours, de lignes de force et de lignes de fracture. Au-delà de la métaphore, les mots se chargent de sens qui diffèrent selon les époques: Sud-Ouest ou Sud Est de la France, Bas-Languedoc, Languedoc méditerranéen ou tout simplement Languedoc-Roussillon. Armand Frémont présent on aurait pu s'intéresser aux problèmes de nomenclature que pose l'existence de deux Normandies, et au passage de la dénomination "basse Normandie " à celle de "Normandie " tout court. Rappelons pour mémoire les anciennes Hautes et Basses Alpes, la Seine Inférieure se maritimisant, la Loire Inférieure devenant Atlantique, des Pyrénées Orientales qui se voudraient bien catalanes, des Côtes du Nord devenues d'Armor. La liste n'est pas close.

\section{Dès lors, comment aborder cette région?}

Dans ses échelles et dans ses champs, selon sa quadruple appartenance actuelle. A partir de ce principe simple et pour n'importe quelle question posée, on peut se demander à quelle échelle géographique on obtiendra une réponse?

Ce qui implique de savoir exactement comment se définissent l'un et l'autre concept et de prendre un exemple.

\section{DEFINITIONS}

- échelle : elle pose le problème de la relation entre un objet et sa représentation, sachant qu'il se passe beaucoup de choses lorsque l'on change d'échelle, lorsque l'on fait varier l'ordre de grandeur d'un phénomène.

- champ : c'est l'aire d'extension d'un phénomène géographique défini par un tissu de relations sur une portion d'espace, ce qui implique une action, des relations, voire des conflits, en tout cas acteurs et stratégies.

\section{EXEMPLE}

21 Un exemple, bien concret, est fourni par l'usine IBM de Montpellier et son installation dans les années 1960 .

Elle répond à :

- une stratégie mondiale, partie de son siège américain? 
- une envie de s'installer en Europe méridionale, pas trop loin de Valencia avec qui fonctionnera un temps une ligne aérienne? peut-être la trace d'une stratégie méditerranéenne dans le domaine de l'informatique civile et militaire restée sans lendemain, à destination des rives sud de la Méditerranée ?

- une réponse à des incitations en France où la firme s'est déjà installée, d'abord à Paris ensuite sur la Côte d'Azur?

- au souci d'un cadre de vie méditerranéen, moins bien que sur la Côte d'Azur, mais mieux qu'en Aquitaine?

- à l'existence d'une vieille ville universitaire garante et porteuse d'enseignement et de culture?

Un peu de tout cela à la fois, plus tout l'inavoué passant par les relais de politique d'influence, de paix sociale, de stratégies de firmes et de collectivités locales.

On passera en revue toutes les appartenances possibles, de la plus petite échelle à la plus grande.

1. Au plan mondial le Languedoc-Roussillon n'est qu'une parcelle du bassin méditerranéen, premier pôle touristique mondial et version européenne du bassin caraibe, avec un peu moins de richesses du côté des paysages, un peu plus du côté de l'épaisseur culturelle. $27000 \mathrm{~km}^{2}$ qui en font l'égal de l'Albanie (28000), de la Sicile (26000) ou de la Sardaigne (24000). Avec comme conséquences deux réflexes, complémentaires et antinomiques à la fois :

- une recherche identitaire, quand le nombre de touristes est supérieur au nombre d'habitants, pouvant aller jusqu'à un rapport de un à dix

- un réflexe de rejet, face au « bronze-cul de l'Europe ».

Il en résulte, au plan des représentations, une assimilation à la Méditerranée alors que la moitié de l'espace régional est montagnard (appartenant à la moyenne montagne), et que la plaine stricto sensu est rare sauf en Lunellois, Biterrois-Narbonnais et Roussillon, là où les lagunes sont comblées. Paradoxe, cette région dite méditerranéenne tourne le plus souvent le dos à la mer, cette région de plaine viticole est pour l'essentiel vallonnée, cette région vue comme basse possède un territoire montagnard très développé. De la même façon, distances euclidiennes et distances « de circonstances » se disputent le territoire, en une série de recompositions « méditerranéennes » :

- l'Est de la Méditerranée que l'on désigne comme « Proche-Orient » est en réalité à $3000 \mathrm{~km}$, donc plus loin que Moscou

- il n'y a que quelques $\mathrm{km}$ entre Sicile et Tunisie, entre Alicante et Oran où l'on parlait pour une bonne part la même langue. Sète et Alger sont à $800 \mathrm{~km}$ l'une de l'autre, soit une distance plus courte que celle que l'on mesure entre Sète et Dunkerque. 
Figure 1 : Les 5 départements du Languedoc Roussillon

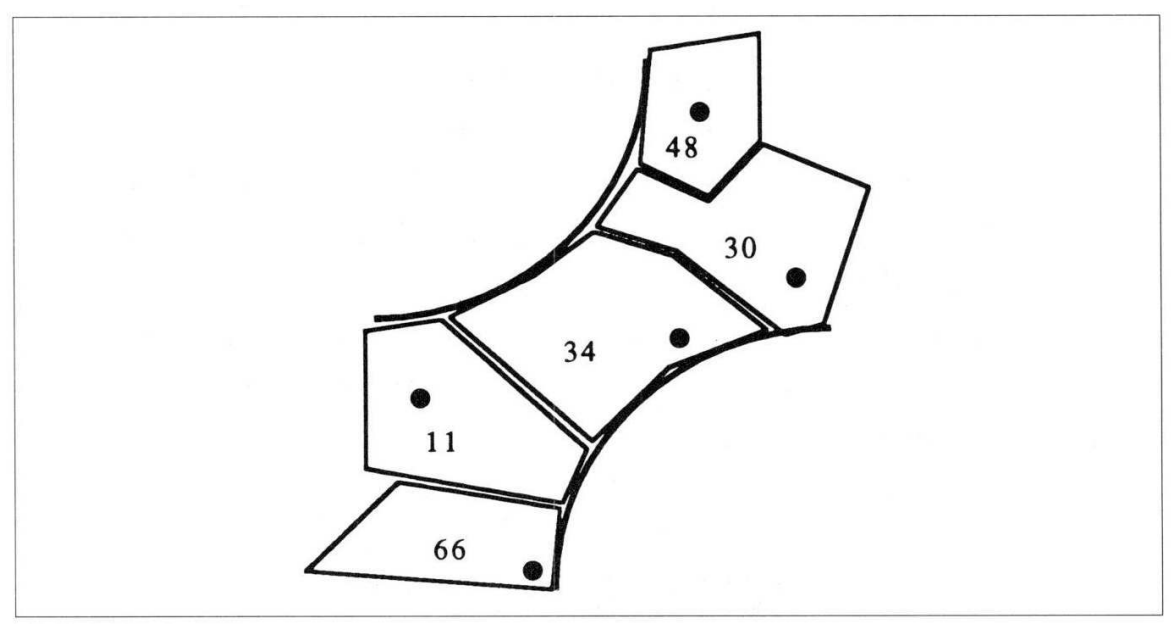
qui va de Londres à Milan, et le Languedoc-Roussillon appartient à ses périphéries, comme pays à la fois du sud de l'Europe du Nord et du nord de l'Europe du Sud. Ce qui le pose doublement en marge,

- dans l'espace, par l'éloignement des centres économiques vitaux,

- dans le temps selon le rythme de la « saison » annuelle, qui n'a qu'une durée réduite entre juin et septembre.

Mais, continuant à relativiser tout cela, on constate qu'il peut aussi être le centre d'une périphérie : soit le nord d'un sud après avoir été le sud d'un nord.

Figure 2 : Le premier pôle touristique mondial : la Méditerranée

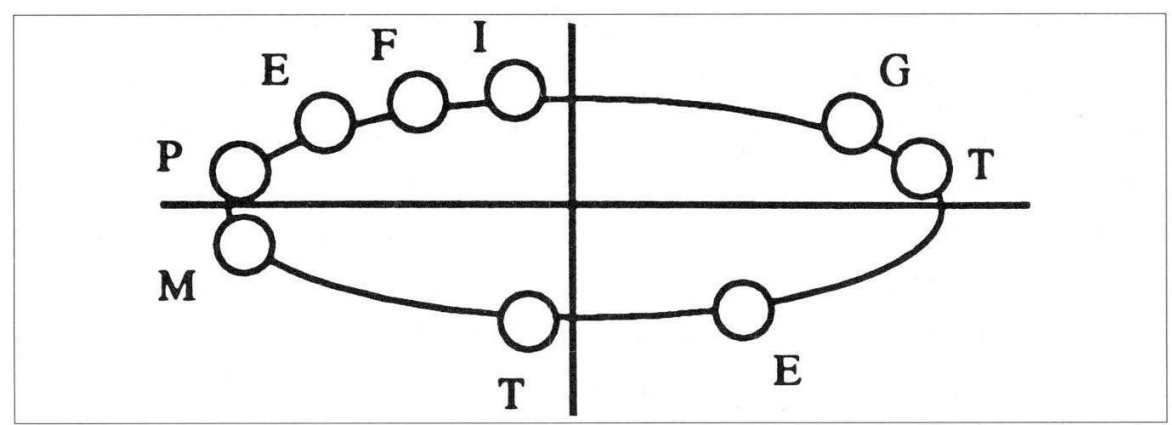
dont les spécialistes notent l'émergence entre le Sureste -en tout cas le Levant- espagnol et la région de Rome -en tout cas la Toscane-, Cette charnière, qui a vu son attrait se confirmer à travers l'héliotropisme, reste en construction mais mérite d'être prise en compte au niveau de la réflexion géographique, tout comme il y a un arc atlantique, un finisterre européen, une mer du Nord.

31 L'avenir est-il au sud ? Dès la mise en place de l'université au XIIIème siècle, à l'évidence ou dans ce creuset où se mêlent Juifs, Arabes, Lombards et Gênois, Provençaux, Catalans, Levantin,... A l'heure actuelle on peut rappeler que $6 \%$ des étudiants étrangers de Montpellier sont libanais, $7 \%$ algériens, $25 \%$ marocains ce qui donne un premier total de 
$38 \%$ pour seulement trois pays (de la Méditerranée). On arrive à $47 \%$ en additionnant Maghreb et Moyen-Orient dans les provenances et à $56 \%$ en ajoutant les pays méditerranéens de la Communauté Européenne et l'Afrique.

Des chiffres à méditer, face à Toulouse, Aix et Nice.

Figure 3 : L'arc méditerranéen

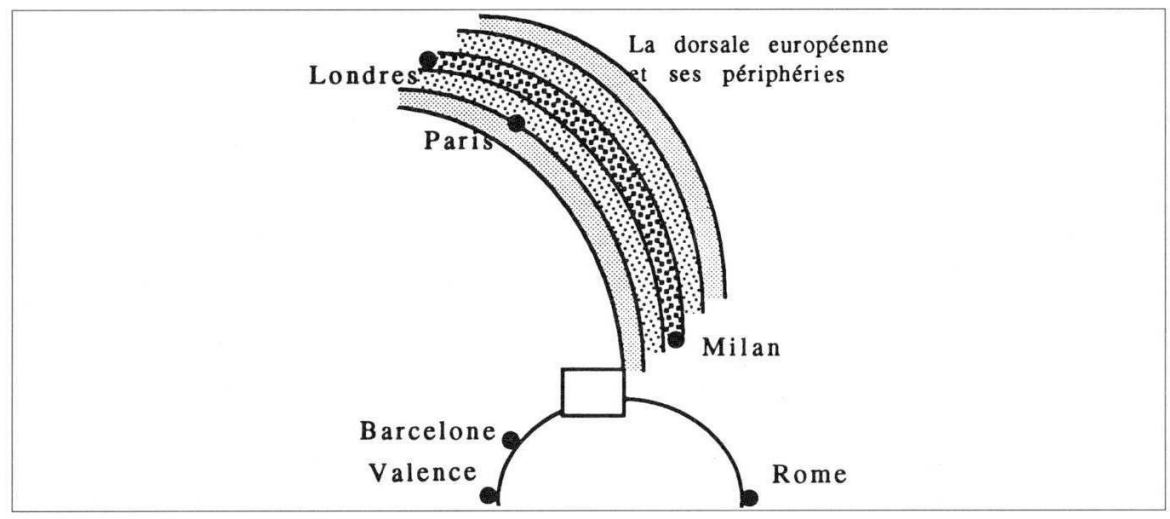
fois qu'on tente de le définir. Vu d'ailleurs, il entre dans un grand ensemble, grand delta ou autre. Dans un sens le sud-ouest peut aller jusqu'au Rhône, dans l'autre le sud-est peut aller jusqu'à la rivière Aude. La Lozère du nord est du Massif central, l'Aveyron méridional se détacherait bien de Midi-Pyrénées, l'Aude occidentale est incontestablement de mouvance toulousaine, quant à la Provence, où est donc sa limite? Au Rhône, au Vidourle, ou aux portes de Montpellier? Un peu des trois à la fois, et l'on s'évertue périodiquement à faire coïncider des limites départementales dont on oublie la fraîcheur et la fragilité avec des césures bien plus anciennes et toujours perpétuées, d'une manière ou d'une autre.

e repartons pas sur des jeux à la fois stériles et révélateurs de faits géographiques hérités. Ou, en d'autres termes, que reste-t-il du Languedoc ? Peut-être quelques arpents de terre "centrés». Ceux qui avaient désigné Pézenas comme capitale des Etats de Languedoc l'avaient peut-être déjà compris au temps où le Prince de Conti accueillait Molière en tournée. Il y a une sorte de Languedoc du Languedoc entre basse vallée de l'Aude et Vidourle, guère plus au delà. Il reste du "vieux » Languedoc quelque chose au cœur de l'ensemble centré comme toujours sur les rives du fleuve Hérault, cette médiatrice entre deux mondes. Avec beaucoup d'immodestie quelqu'un a dit que Poquelin était né à Paris et Molière à Pézenas ; avec quelque humour Roger Brunet aime à rappeler qu'ici est né Paul Vidal de la Blache, fondateur de la géographie régionale, au cœur de sa région. 


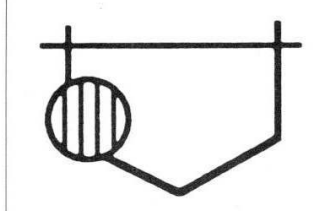

aquitain:

vignoble

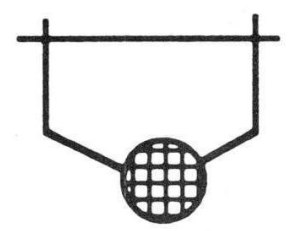

du Languedoc Roussillon:

vignoble et tourisme

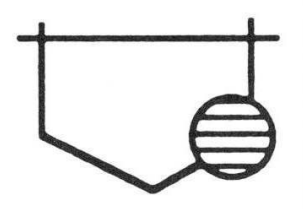

provençal:

tourisme

\section{Méditerranée.}

Figure 5

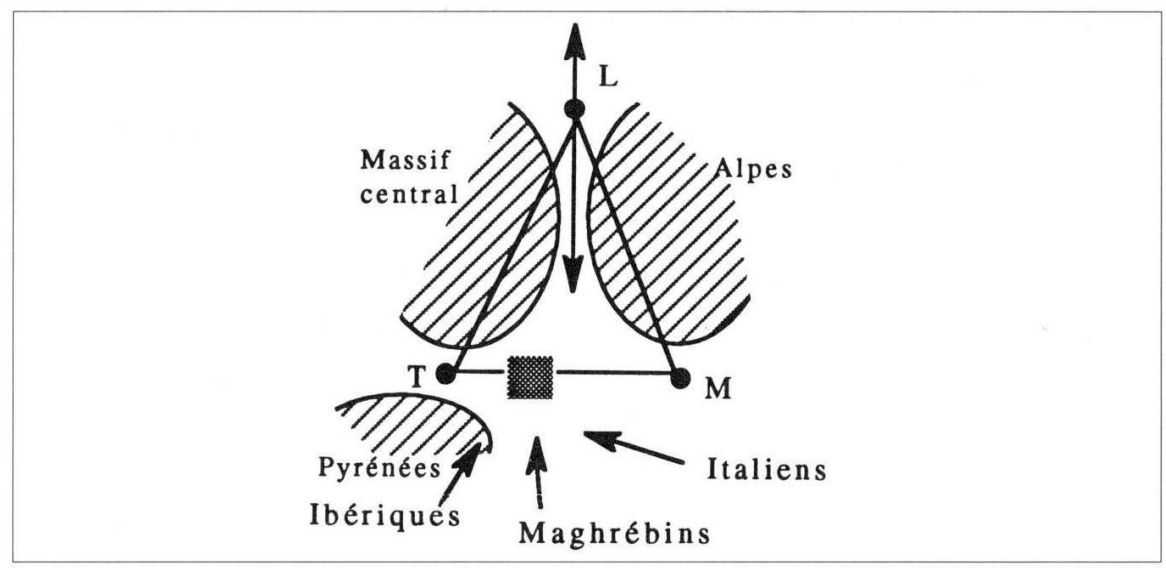

\section{La spécificité géographique actuelle du Languedoc-Roussillon}

Trois thèmes rendent compte à la fois d'une spécificité géographique et de mutations en cours. Ils ont l'avantage d'offrir quelques réinterprétations des composantes traditionnelles à travers quelques pistes qui seront simplement évoquées :

\section{Les hommes.}

40 Croissance et records de flux sont évidents, mais pour un contenu différent. 2 millions d'habitants se répartissent pour l'essentiel (le tiers) entre Sète et le Rhône, et le dixième du total sur la seule ville de Montpellier. Après une déprise accélérée l'arrière-pays semble pour une grande part stabilisé, même de façon un peu diluée. Entre la plaine littorale et le reste du territoire, il y a moins d'oppositions que ne le disent toutes les dérives déterministes, la population se concentre là où il $\mathrm{y}$ a déjà le plus d'habitants, dans l'est. Depuis la deuxième 
guerre mondiale on enregistre des constantes, selon les différents secteurs d'activité :

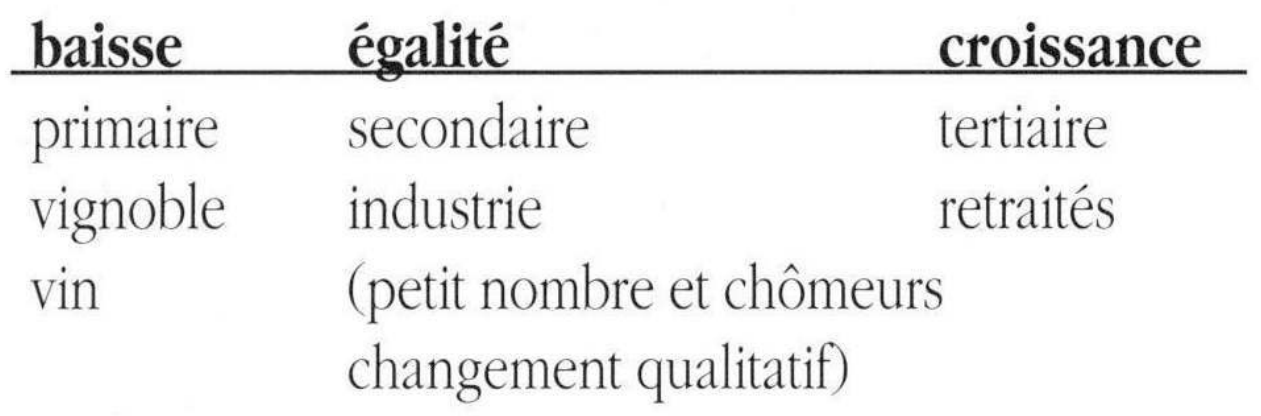

41 Les leaders sont en perte de vitesse, COGEMA, IBM et Perrier, les trois à plus de 2000 emplois, devant Carrefour et Casino, défraient de plus en plus souvent la chronique journalistique. La réalité passe par la présence de 200000 fonctionnaires, le dixième de la population totale, dont 35000 pour l'éducation nationale, 25000 pour le secteur hospitalier, les mairies, les industries de pointe comme informatique et secteur biomédical.

Population et activités soulignent de nouvelles dynamiques, une pâte de fortes densités sur le passage des deux couloirs (audois et rhodanien) plus la rocade littorale, renforcées sur les villes avec une diffusion autour des agglomérations.

\section{Le système viticole.}

Omniprésent encore, le vignoble de masse recule dans les bas-fonds, alors que grimpe la qualité. Marsillargues produit désormais des légumes, des asperges ou des fraises. On comptabilise une liste de 43 vins de pays, de « côteaux » ou de « côtes » avec souvent des noms d'anciens pays, et une consommation soucieuse de choix. Au sommet AOC et VDQS, avec les blanquettes de Limoux, quatre muscats, les Côtes du Rhône, Corbières et Minervois. En qualité apparaissent sur la carte les confins rhodaniens et l'ensemble AudePyrénées-Orientales. Le reste est en pleine reconversion, plus ou moins avancée. Mais au plan national trois simples chiffres soulèvent quelques problèmes, le LanguedocRoussillon est au premier rang français en étendue avec $38 \%$ du vignoble, $42 \%$ de la production mais seulement $20 \%$ de la valeur (7 milliards de francs).

Ce que montre un schéma de système, avec ses différentes entrées, c'est la nécessité de revoir la vigne d'une autre façon, elle qui n'est plus la principale production agricole de la région, venant désormais après le tourisme.

\section{Le tourisme.}

Trois espaces en bénéficient, pour l'essentiel :

le littoral avec 200000 places

la montagne : les Pyrénées et les Cévennes

entre les deux les garrigues et la moyenne montagne, assez vides en apparence mais comptant de nombreuses résidences secondaires. Par exemple, 6000 places pour la seule commune de La Salvetat. Mais un peu comme pour les vins de consommation courante, le tourisme de fréquentation courante ne doit pas empêcher de découvrir d'autres crus, que connaissent bien les autochtones, et que l'on se garde un peu d'offrir au touriste tout 
venant. Les guides ne disent pas tout. Le rapport entre accueil et résidents est toujours supérieur à $200 \%$, atteignant $1000 \%$ sur le littoral en saison.

Figure 6 : Station littorale ancienne

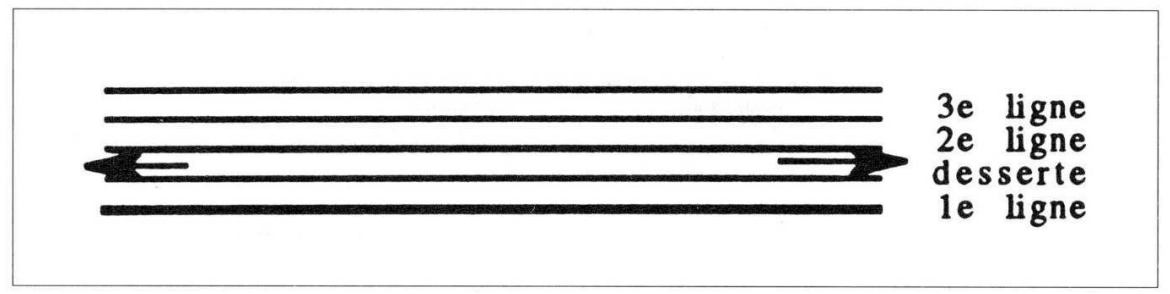

Figure 7 : Station nouvelle, l'empilement de la première ligne dans la troisième dimension

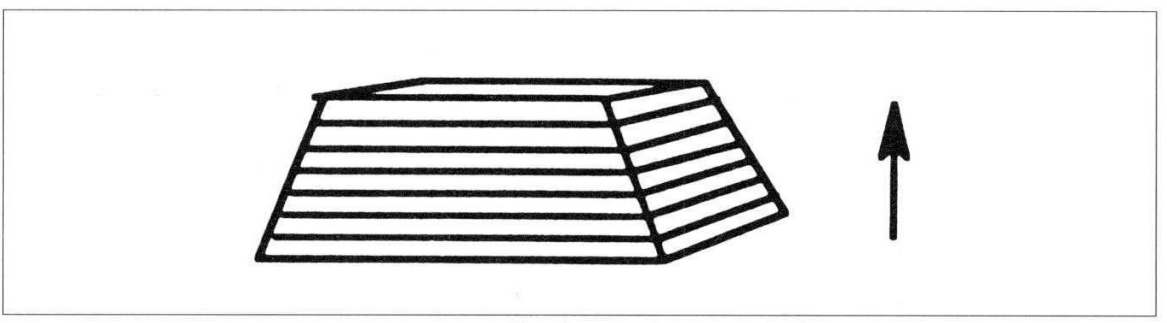

Figure 8 : La marina, la démultiplication du front d'eau dans les terres

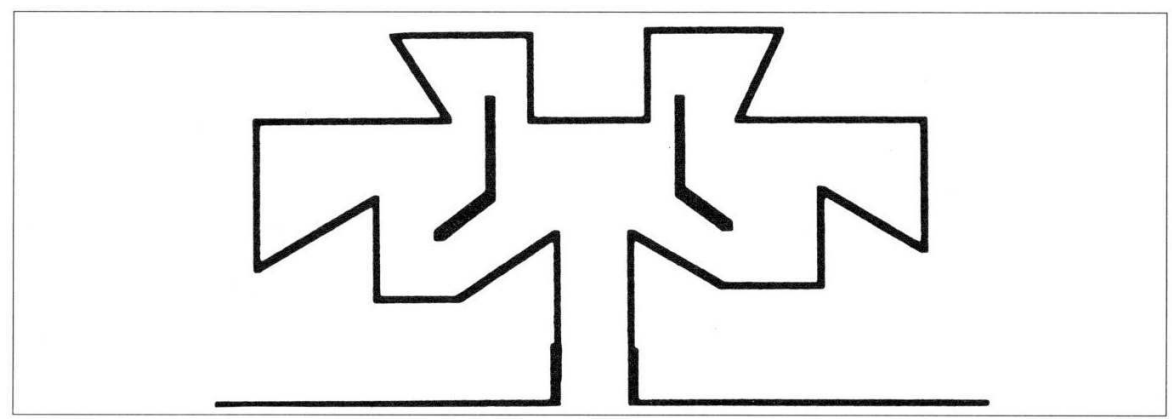

Il faut rappeler quelques dates, simples et récentes :

- 1963, sous l'autorité de la Mission interministérielle, mise en place de six unités touristiques : Grande Motte, Cap d'Agde, Gruissan, Leucate-Barcarès, Canet-Saint-Cyprien ; la dernière, celle de l'embouchure de l'Aude étant provisoirement abandonnée avant d'être reprise ultérieurement par le Conseil Général.

- 1970, Parc National des Cévennes

- 1973, Parc Régional du Haut-Languedoc

- 1977, Pays d'accueil

- 1979, Zone littorale définie avec un conservatoire, en 1985, dont l'autorité s'étend sur 67 communes.

L'éventail est donc au complet, de la mer, de la montagne, et de l'espace " intermédiaire ». Voilà la réalité, mais toute une partie mythique l'emporte sur les réalités, à base de soleil, de vacances. En fait parler de tourisme en Languedoc-Roussillon revient à deux choses pour l'essentiel, l'utilisation du front d'eau, les recompositions touristiques selon le temps et des stratégies différentes (l'image donnée est variable). 
Le front d'eau c'est l'utilisation de la première ligne, celle qui se commercialise le mieux, selon trois grandes étapes récentes :

- anciennement le désir d'accès direct à la plage et la vue sur la mer qu'il propose et qui donne ces stations étirées, parallèles au front d'eau qui finissent par se rejoindre comme Palavas et Carnon, avec ensuite une deuxième ligne, une troisième lorsque la seconde est saturée, ... selon un « urbanisme » de la linéarité, sans profondeur.

- ensuite le passage à la troisième dimension que procure la Grande Motte où la première ligne, en tout cas son succédané, est empilée en étages successifs formant pyramides. Il suffira ensuite de justifier cet empilement et l'on aura recours à l'imitation des Cévennes dans les lointains de l'arrière-plan, à la garantie du maximum d'ensoleillement ou au résultat d'expériences en soufflerie. Au choix, sans que soit jamais dit que l'immeuble en hauteur est encore le meilleur moyen de valoriser un espace au sol.

- enfin la dernière étape est celle de la marina, ou -sous le thème antithétique du retour à « l'échelle humaine »- l'introduction d'un front d'eau artificiel ménagé à l'intérieur des terres à partir d'un goulet très étroit qui préserve seulement la communication avec la mer. Les recompositions touristiques apparaissent sous le thème des éléments naturels, toujours les mêmes, le soleil, le vent, dont on fait un usage différent au gré des époques, voir les travaux de Christine Maurin. Le vent est salvateur, quand il vidange les lagunes ou les graus, quand il chasse les moustiques. Il souffle dans les dépliants publicitaires selon que les sports de voile sont mis en avant ou pas depuis l'Antiquité.

\section{Les dynamiques spatiales}

On rappellera quelques définitions, de modèle, chorème, qui autorisent une certaine relecture des dynamiques spatiales.

Les chorèmes sont des structures spatiales simples dont la combinaison donne des modèles d'organisation de l'espace parfaitement opératoires pour en saisir les dynamiques. Les dynamiques soulignent un changement résultant d'un jeu de forces. La dynamique des territoires étudie les changements des organisations territoriales et les forces qui les provoquent. Des champs en résultent.

Le modèle proposé il y a peu (M. Clary et al. Cartes et modèles à l'école)combinait 6 chorèmes réunis deux à deux pour proposer une explication du Languedoc-Roussillon : un CADRE...

- l'implantation urbaine et le vieux passage

- l'économie ancienne et les stations touristiques

- des densités contrastées et une dissymétrie est-ouest ...

- sa VALORISATION...

- un couloir urbanisé

- une évolution économique

- des déséquilibres ...

- une SYNTHESE

- le modèle final du Bas-Languedoc révèle un certain nombre de disparités régionales, au milieu des années 1990. On peut procéder un peu différemment, de façon plus synthétique, sans remettre en cause l'essentiel de ce qui vient d'être montré. 


\section{Les oppositions.}

Une approche dichotomique donne un inventaire raisonné qui passe par :

- les vides et les pleins, une pâte périurbaine dense

- le couloir et « le reste »

- le faisceau des villes littorales et celui des villes de piémont

- un carrefour et des zones mal innervées

- des densités élevées et des déserts humains

- un vignoble et une absence de vignoble.

\section{Les écartèlements et complémentarités,}

ou un recentrage en cours, mettent l'accent sur des écartèlements socioculturels :

- aquitain à l'ouest avec l'ancien réduit cathare

- provençal et rhodanien à l'est

- montagnard au nord, le désert huguenot inclus

- catalan au sud (1669, paix des Pyrénées)

Ce que matérialisent les tombées urbaines de Toulouse, de Marseille, ou -bien plus réduites- de Barcelone.

Figure 7 - Oppositions : vides et pleins ou « le couloir et le reste " avec deux faisceaux de villes dont un de piémont, un carrefour des densités élevées, sur un vignoble

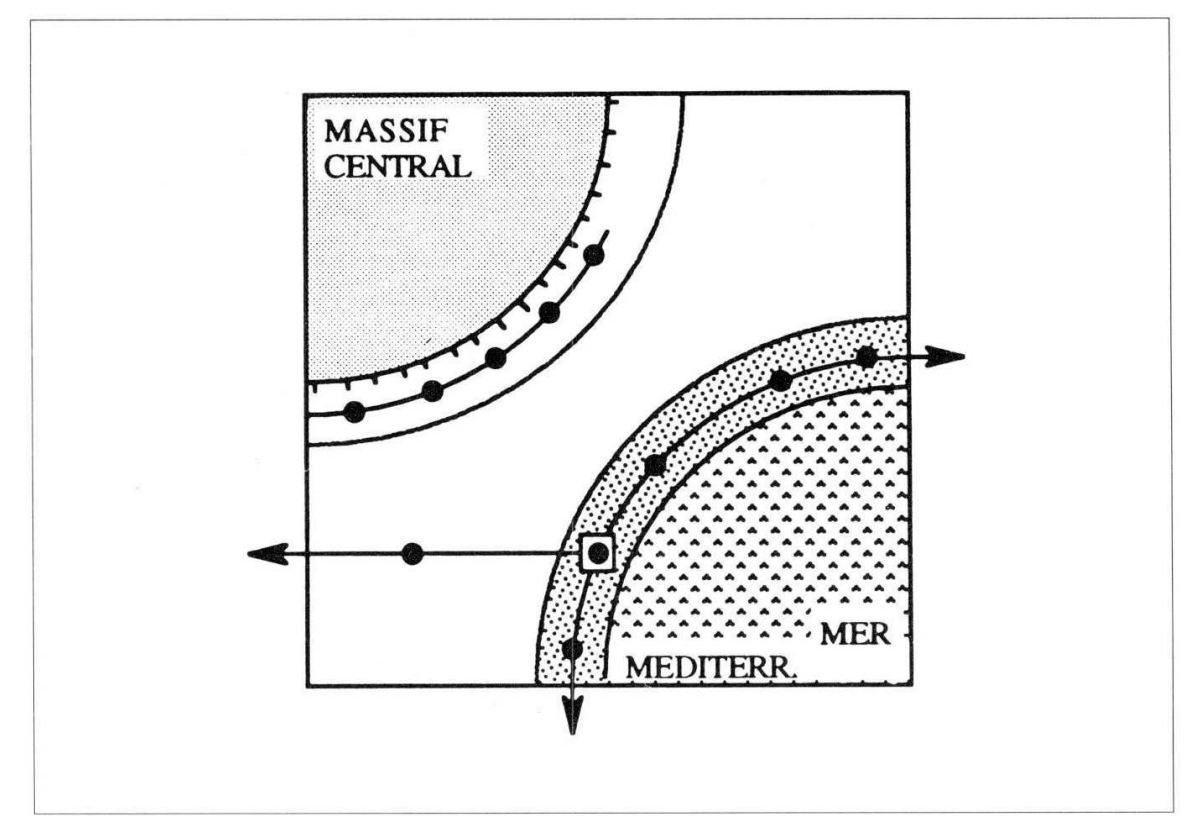


Figure 8 - Ecartèlements et complémentarités ou un recentrage? Ecartèlements culturels : Aquitaine W, Provence $E$, Montagne $N$, Catalogne S Complémentarités : hommes et produits, plaine-montagne

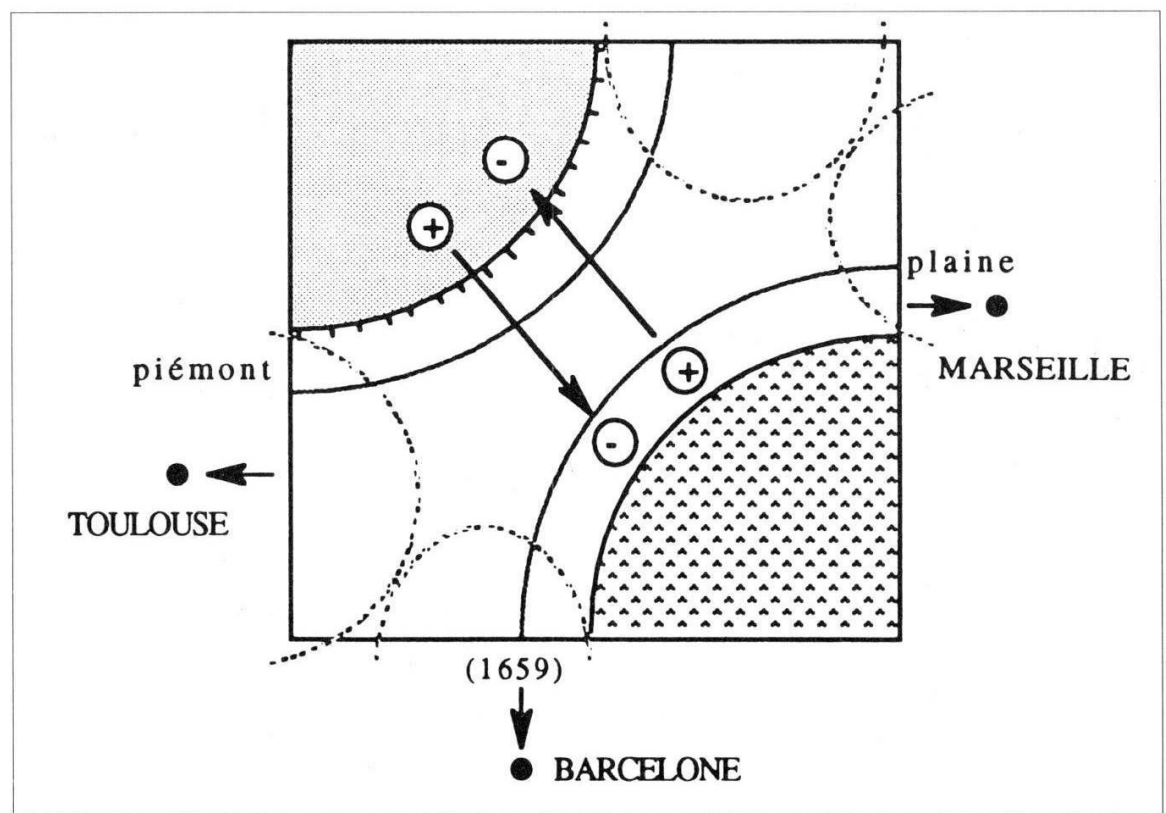

Figure 9 - Dynamiques actuelles ou une opposition nord-sud à une opposition est-ouest. Naissance d'une capitale régionale dépassant les tombées urbaines d'origine que souligne le modèle. Naissance d'un littoral

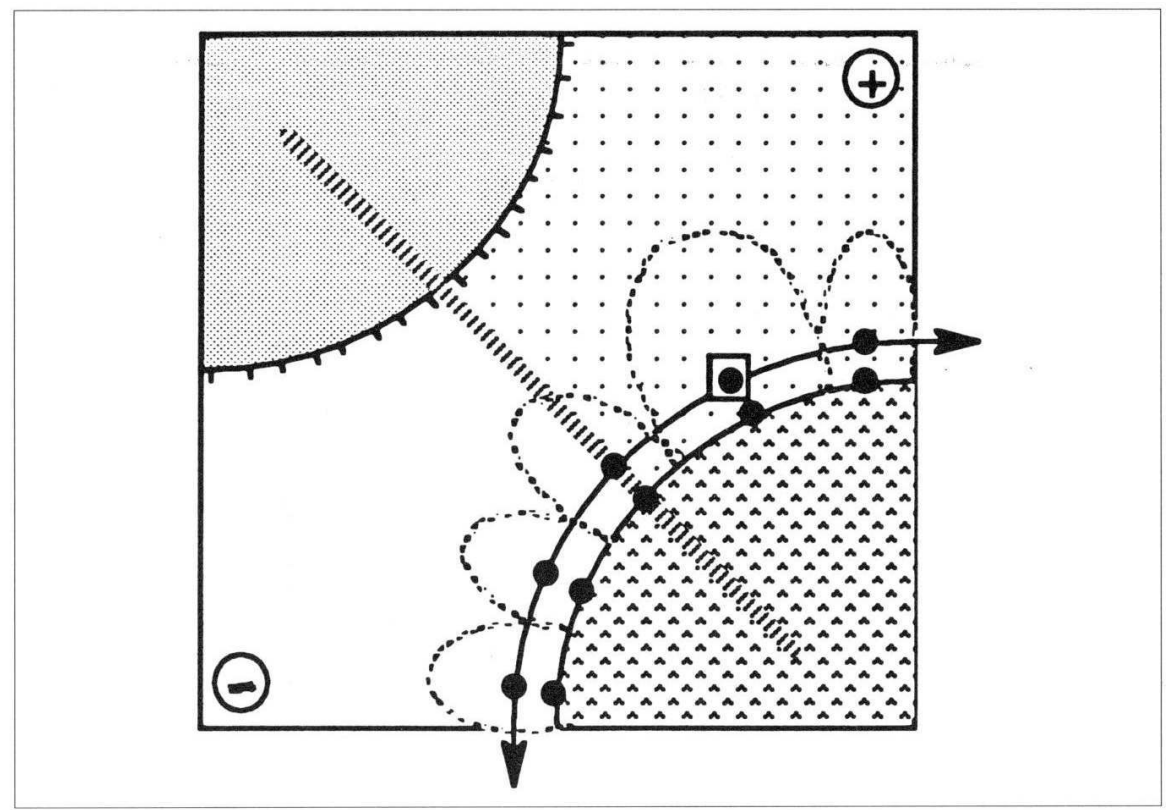




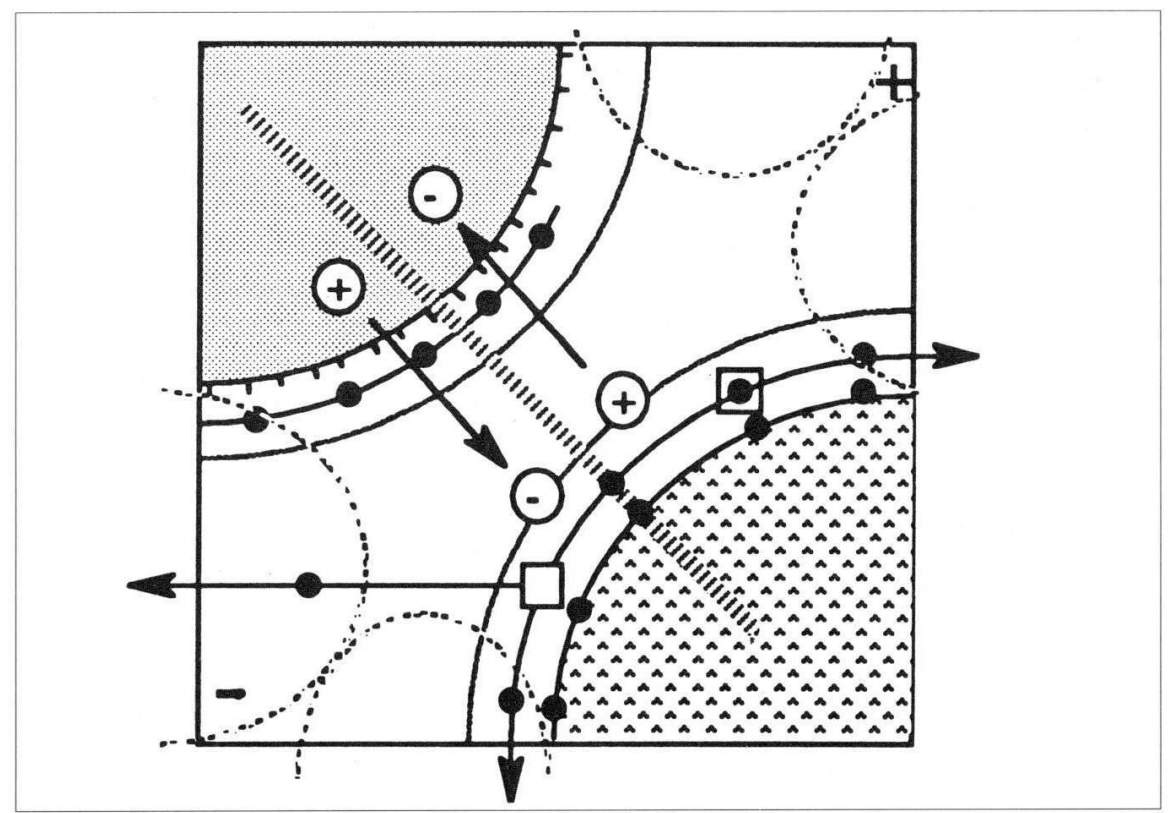

Des complémentarités anciennes que l'on dirait volontiers pseudogéographiques parce que plus présentes dans une mémoire collective qu'une réalité, entre montagne et plaine :

- hommes et produits,

- sel et vin contre céréales et merains,

ce que traduisent les pénétrantes ferroviaires vers le Centre à partir du couloir circumlittoral commun.

\section{Les dynamiques actuelles.}

D'une opposition nord-sud on glisse vers une opposition est-ouest, à travers la naissance d'une capitale régionale dépassant la marqueterie urbaine des origines sans ville nettement prééminente. Et suit la naissance d'un littoral, allant d'initiatives indigènes au $\mathrm{XIX}^{\mathrm{e}}$ siècle (station de Palavas-les-Flots) à des décisions d'origines exogènes au XXème.

\section{La synthèse.}

60 Le terme est mal choisi. Elle ne diffère guère de la précédente, à quelques années de distance, mais n'est peut-être plus possible. Plutôt que de synthèse on parlerait d'état des lieux, de bilan spatial à un moment donné. A l'heure actuelle, pour dépasser le stade des partitions détaillées ci-dessus on dirait qu'une bonne part d'explication de l'organisation spatiale de la région passe par une formule abrupte mais simple, on dirait volontiers « Montpellier et le reste ».

\section{Conclusion}

61 Cinq ou six choses que l'on commence à savoir d'elle.

62 1. S'il faut absolument présenter la région selon ses partitions actuelles, on peut retenir comme entités le Roussillon, un vieux Languedoc à l'ouest, un néo-Languedoc à l'est, la 
montagne lozérienne en un écartèlement plus proche de la réalité spatiale que la bipartition habituelle. Un cinquième ensemble n'atteint pas tout à fait le stade de l'individualisation, le littoral. Cela n'est pas abordable dans l'absolu, mais à replacer à d'autres échelles, dans d'autres ensembles.

L'arc méditerranéen devient peu à peu un axe structurant, appuyé depuis les années 1930 sur une concentration de population, l'extension des périphéries urbaines jusqu'au continuum. On a commencé à parler du duopôle Montpellier-Nîmes, le doublet NarbonneBéziers suit, d'autres viendront.

2. On était parti d'une ville première, Narbonne en Narbonnaise puis plus simplement en Narbonnais, on revient à une autre forme d'émergence bien plus tardive et dénommée seulement pour la première fois en 985 , Montpellier. Entre temps se sont maintenus ou sont passés le temps de Sète, d'Agde, de Nîmes, de Pézenas, d'Alès, de Béziers, de SaintPons, selon des hiérarchies et des époques différentes. Sans parler de Lodève dont on ne parle guère, au centre de son territoire exsangue, ou de Ganges en son piémont. Toutes les villes de la région, à un moment ou à un autre, ont fonctionné ou fonctionnent ou fonctionneront comme des révélateurs, marquant toutes le pas à moment donné au cours $\mathrm{du} \mathrm{XX}^{\mathrm{e}}$ siècle. A deux exceptions près, en une taille et un poids non comparables, Montpellier et Narbonne dont les courbes de population -toutes proportions gardéessont toujours ascendantes.

3. Le Languedoc-Roussillon est parfois un sous-produit et un relais à la fois de la Côte d'Azur. Plus sèchement on mettrait l'accent sur la notion d'ersatz, à l'échelle méditerranéenne, maillon qui n'a pas été évoqué en tant que tel jusqu'ici. Ce qui peut sembler dévalorisant, mais a l'avantage de remettre les deux ensembles en situation. Montpellier sous-Nice, Côte Vermeille sous Côte d'Azur (peintres y compris), Sète sousMarseille (loin derrière), Grande Motte plagiant Marina baie des Anges, il y a un peu de vrai dans tout cela. Quelques qualités climatiques en moins, quelques avantages dans les tractations foncières en plus.

4. En forme de hit parade, mais largement revu et corrigé, tout triomphalisme mis à part.

- Pour le tourisme la région est au premier rang en France, mais pour ce que l'on dénomme désormais hôtellerie de plein air, elle est au troisième, après PACA et Rhône-Alpes.

- Pour le vin, ce qui assure un classement en tête c'est plus la masse que la qualité, même si celle-ci s'affirme.

- Pour les mouvements de population, les totaux masquent le retour d'anciens Languedociens ou l'arrivée de retraités, dans les deux cas une population âgée : forte charge de retraités, femmes sans activité.

- Pour l'économie, les records de morcellement et de pluriactivité persistent chez les agriculteurs. Dans les implantations industrielles beaucoup de créations se soldent par beaucoup de disparitions. La région continue à se tertiariser, mais est-ce un signe de bonne santé économique ? La formule relève du journalisme parce que le trait est forcé, il y a ici un côté « sun belt » pas encore « Silicon valley », pour parler « américain ».

- Pour le PIB le Languedoc-Roussillon est dans les dernières régions de France. La misère sous le soleil, comme en pas mal de pays au sud?

5. L'évolution montre une série de phases de compositions, décompositions, recompositions de la région sous l'influence de dynamiques urbaines, de productions économiques qui résument quelques décennies et débouchent sur un recentrage sur Montpellier. Le débat de fond n'est pas là, entre un choix montpelliérain et une organisation multipolaire, car la capitale régionale bénéficie à l'ensemble, comme on ne 
le pense guère, en certains cas à Perpignan, ou en d'autres chefs-lieux encore moins connus. Perpignan certes, mais aussi à Mende, Carcassonne ou même Nîmes, pour des raisons différentes. Le dire c'est aussi sortir d'une géographie pseudo-aseptisée pour prendre en compte des réalités politiques qu'il faut bien voir en face. De la même façon le monde universitaire c'est la vieille place de Montpellier et pas telle ou telle place récente, dotée d'IUT ou autre. Une question d'échelle. De la même façon le monde économique méditerranéen ne doit pas perdre de vue que Barcelone et Montpellier ne "jouent » pas dans le même espace, la première nommée jouant dans la cour des grands.

D'où, en fait, l'existence d'une société duelle qui rapproche ici le plus grand nombre d'adultes bacheliers et le plus grand nombre de sans diplômes, le plus large éventail d'accueil en termes de nouveaux arrivants et le plus de chômage. Un LanguedocRoussillon désormais au dernier rang pour la natalité, la fécondité et donc le solde naturel : on n'y fait plus d'enfants, comme en Espagne, comme en Italie, chacun en ce domaine se « déméditerranéisant ».

Les vieux manuels, en retard d'une guerre, et quelques autres moins vieux y perdent leur savoir, découvrant quelques nouveautés, et quelques contradictions entre territoires normés et mémoire territoriale. Il y va de quelques révisions, certainement pas déchirantes, et surtout de la crédibilité d'une discipline qui en vaut la peine. Le reste suivra, remettant à leur place les pièces de l'ensemble.

\section{Dominique Borne}

L'historien que je suis est-il capable d'apporter un autre regard? Un historien qui a cependant beaucoup appris dans la thèse de Raymond Dugrand, un grand livre de géographie.

Je me limiterai à quelques remarques : Robert Ferras est le seul, mais comme à regret, et ne pouvant s'en empêcher, à avoir évoqué des paysages. Il l'a fait très vite au début de ce qu'il nous a dit alors que dans les deux leçons précédentes nous n'avons pas eû trace d'évocation de paysage.

Deuxième remarque.

En pédagogie il est nécessaire pour qu'un auditoire comprenne qu'il s'agit de clichés, que l'on présente d'abord ces clichés. C'est une illusion de l'enseignement de dire qu'il faut détruire les vieux clichés sans les décrire. Parce que pour les élèves, ces clichés sont neufs. En géographie comme en histoire il faut analyser les clichés avant de les démonter. Partir des vieilles images du Languedoc est donc fondamental, on ne peut pas ne pas faire ce détour.

\section{Troisième remarque}

Quant à l'usage des chorèmes, je ne peux plus écouter un géographe sans entendre qu'une périphérie devient centre ; l'exemple des Etats-Unis où le centre devient périphérie, et la périphérie centre. Je l'ai vérifié pour le Languedoc, il était inévitable, le patriotisme local aidant, que cette périphérie devienne centre.

Question : A enseigner le Languedoc-Roussillon à des élèves de différents âges mais qui l'habitent je me demande pourquoi on ne fait jamais appel à leur regard personnel. Il n'y a pas de géographie égocentrée, qui parte de leur vision à eux et pas seulement des représentations qu'on leur propose. 
76 On compte deux niveaux très différents: les représentations des élèves et les représentations qui traînent à la télévision, dans la presse... ce qui n'est pas la même chose. Ce qu'on leur demande, souvent, ce sont des représentations médiatisées mais pas forcément leur vision. R. Ferras est le seul à avoir parlé de l'autoroute, là où l'on voit les camions espagnols... L'enseignement de la géographie ne doit-il pas rendre les élèves capables de comprendre ce qu'ils voient en sortant du cours de géographie, et de dire : je vois un camion espagnol sur l'autoroute, ça me dit quelque chose sur la région mais aussi sur sa place en Europe. On est passé d'une géographie où l'on partait de diapositives, c'est-à-dire d'images ou de paysages à une géographie où l'on part de modèles. Si bien que l'image, en géographie, est devenue démodée.

Certes il y a de mauvais usages de la diapositive. J'ai vu un enseignant voulant prouver que l'Asie était très peuplée, montrer une rue populaire de Pékin. Ce qui ne prouve pas que l'Asie soit peuplée. Ce type de diapositive est absurde. Mais il y a un autre usage, une éducation du regard des élèves. La géographie ça sert à mieux voir le Monde, voir au sens précis de ce mot et à réagir à différentes échelles. L'exemple banal du camion sur l'autoroute est à reprendre : le camion, je le vois, mais il permet de penser à une autre échelle que celle de l'autoroute et donc cette image facilite le passage d'une échelle à l'autre.

Deux remarques encore :

79 Le mot «dynamique » me gêne quand il qualifie des espaces. «Les plus dynamiques: l'Hérault... les moins dynamiques : la Lozère... etc ». Pour des élèves le mot est connoté positivement. Même si pour des géographes il ne l'est pas obligatoirement.

Peut-on se référer à une géographie de la manière de vivre et d'habiter ? Hier et aujourd'hui on n'a pas vu ces bourgs urbains, ce pays plein de villes souvent antagonistes.

Pour finir, je vous propose une lecture sur le Languedoc-Roussillon : «Ce couloir, où voie romaine et route royale, canal et chemin de fer se pressent, est un passage de peuples. Sans doute il y eût toujours des relations fondées sur la nécessité des échanges entre la plaine et la montagne. Ces rapports, menus et de détail, issus de contrastes rapprochés, jouent dans la vie du Midi un rôle dont il faut tenir grand compte. De Narbonne à Toulouse... grande voie historique foulée par les Gaulois Tectosages, les Romains, les Wisigoths, les Arabes, les Croisés de Simon de Montfort, les Anglais du Prince Noir et ceux de 1814, beaucoup de bourgs, de petites villes jadis fortifiées, se distinguent de loin sur les premières croupes de la Montagne Noire, sur les côteaux de Saint-Félix et sur les mamelons qui bordent la plaine, ils sont les témoins des temps de luttes, les survivants d'une vie qui s'éteint et dont les foyers se déplacent ».

L'Auteur? Vidal de La Blache dans son Tableau de la géographie de la France.

\section{Pierre Desplanques}

Je voudrais vous dire le plaisir que j'ai à me trouver dans ce colloque, au moins pour deux raisons. La première, c'est qu'il est le prolongement et le relais supplémentaire du colloque tenu à Amiens il y a deux ans, qui mettait en perspective un certain nombre d'évolutions que vous avez contribué à dessiner et à porter jusqu'au cœur des classes.

La deuxième satisfaction est plus intellectuelle et tient à l'affirmation qui doit être sensible dans tous les établissements, qu'il existe dans l'Education Nationale un axe 
majeur intellectuel, construit sur des savoirs, qui associe l'université, les professeurs, les inspections générales et régionales. Cet axe majeur lié à une formation commune que nous avons tous reçus à l'université, pénètre jusque dans les classes pour nourrir les esprits dans toutes les grandes disciplines de connaissances.

Cet axe intellectuel peut être quelquefois masqué par un autre axe, celui de la gestion. Nous sommes bien entendu tous sous l'obligation de tenir les ressources dont nous disposons pour en faire le meilleur profit pour l'enseignement. Cet axe prend parfois le pas sur l'axe pédagogique et, dans cette ambiance de rénovation des lycées qui a débuté l'an dernier et pour laquelle les professeurs d'histoire et géographie ont été pleinement dynamiques (au sens plein dont on parlait ce matin). Je crois que dans cette rénovation qui va entrer en classe de première nous avons besoin de réfléchir aux processus d'apprentissage des élèves mais aussi à la connaissance que nous devons porter dans les classes.

Pour entrer dans le débat je voudrais user de mon privilège de président: se poser la question de l'enseignement de la géographie, certains diraient des géographies, impose bien sûr de réfléchir au rôle que joue cet enseignement par rapport au monde qui évolue. C'est une banalité que de le dire mais avec une conséquence immédiate: l'objet de la géographie n'est pas stable. Celui de l'histoire l'est-il d'ailleurs? Certes, cet enseignement est conservateur d'un savoir établi, légué par la tradition universitaire et scolaire, mais il intègre aussi la nouveauté du monde, en mouvement, en utilisant toutes les ressources dont l'homme dispose, y compris les moyens modernes d'information et de communication.

La géographie n'a pas pour fonction de dire seulement ce qui est, elle doit situer l'élève dans la prospective, pour que le travail dispensé aujourd'hui ne soit pas mort demain. C'est ce que Roger Brunet ce matin appelait prendre du recul, j'ajouterai volontiers: prendre du recul pour mieux comprendre l'avenir.

On est assuré de comprendre l'avenir si on connaît ce qui est. Tout en étant conservatrice, la géographie donne à l'ensemble des élèves les moyens intellectuels et les connaissances qui permettent de comprendre le monde actuel et celui de demain.

Elle ne s'arrête pas dans ses ambitions, au présent. Plus simple à exposer qu'à faire, dans des classes qui sont hétérogènes, c'est la raison pour laquelle nous avons essayé de nous éclairer par des témoignages, des analyses, de nos amis européens, d'Italie, d'Espagne, de Grande-Bretagne. Cela devrait nous permettre de mieux cerner les positions intellectuelles qui donnent aux élèves la capacité de saisir les évolutions, de comprendre les moyens et les fins de l'action humaine.

90 Nous allons donc entendre trois exposés, avec tout d'abord la parole à Monsieur Tony Male, directeur du Central Bureau dont la fonction est d'organiser les actions du Ministère de l'Education Nationale au niveau international. Pas un exposé classique de géographie mais portant sur un nouveau système éducatif.

\section{Tony Male}

91 "J'espère que vous me pardonnerez si je vous parle en français. Il m'aurait été beaucoup plus facile de vous décrire les principes qui gouvernaient l'enseignement de toutes les matières dans nos écoles d'état, c'est à dire les neuf-dixièmes, il y a quelques années, bien qu'impossible de définir le contenu des programmes. A cette époque, et jusqu'en 1988, les 
établissements définissaient leurs programmes eux-mêmes, sous l'égide administrative, mais pas le contrôle, des Local Education Authorities (LEA), qui géraient l'administration, les bâtiments, et veillaient sur les conditions de travail des professeurs. Il reste à noter que les chefs d'établissement choisissaient leurs propres professeurs eux-mêmes, après interview, et le font toujours. Le Ministère (le Department of Education \& Science DES) n'exerçait pas d'influence directe sur le programme. C'était «un système national localement administré » (anglicisme, bien sûr).

Etant donné qu'il y avait bien plus de cent Local Education Authorities rien qu'en Angleterre et au Pays de Galles, nous possédions, avec les Américains, un des systèmes les plus décentralisés du monde. Une fragmentation qui voulait que même à l'intérieur de la salle de classe le professeur exerçat un contrôle personnel sur le contenu des programmes dont il ou elle était responsable. D'un côté c'était très bien - j'y ai passé quatorze ans de ma vie.

93 A ajouter qu'il y avait un choix de Boards qui « offrait » des examens agréés par l'Etat à 16et à 18 ans et des différences de rythmes de scolarité entre des Counties et leurs LEA (mêmes s'ils étaient parfois contigus) et vous avez compris que nous vivions un système, ou plutôt des systèmes qui engendraient des essors extraordinaires, et un développement d'individualités fascinant au niveau de l'établissement, des professeurs et de leurs élèves, mais qui présentaient de grands désavantages au niveau de compatibilité, harmonisation et transfert, sans oublier le côté contrôle.

94 Tout ceci a duré jusqu'en 1988 moment où THE EDUCATIONAL REFORM ACT (ERA) est entré en vigueur. Il y a eu de nombreuses modifications depuis dont un White Paper (projet de loi) tout récent. Actuellement donc, en Angleterre et au Pays de Galles nous traversons la période de changement la plus profonde que nous ayons jamais connue dans l'enseignement. J'emploie volontiers le verbe au temps présent car il y a tant de résistance parmi les professeurs - chargés du déroulement pratique des examens aussi bien que du contenu des cours, bien sûr - que je ne suis pas persuadé que les Réformes notamment en ce qui concerne le fameux « testing »- puissent se dérouler en respectant l'agenda établi.

95 «I am jumping the gun» (faux départ). J'ai choisi de décrire ce qui se passe dans l'organisation de l'enseignement car je crois qu'il est évident que ceci définit le climat psychologique de l'apprentissage des matières et la perception, chez les élèves, de leur importance respective.

96 Déjà l'Histoire et la Géographie se présentent dans les salles de classes partout au Royaume Uni commes disciplines séparées (bien que liées). Ceci a toujours été le cas. Je n'ai pas l'intention d'entrer dans le détail de la composition des programmes mais si vous me laissez votre nom et l'adresse de votre institution je vous communiquerai les programmes dans le courant du mois de juin. Je pense que c'est justement le cadre, l'environnement, qui, vu de l'extérieur, a peut-être besoin d'interprétation. Nous avons, même maintenant, un système très " déconcentré " pour beaucoup de choses. Je dirai même que le degré de décentralisation s'est accru avec la lente « dissolution » des LEA et toute cette panoplie d'autres réformes menées par le gouvernement et qui vise le gouvernement local en particulier. Les effets sont clairement visibles au niveau de l'administration des établissements scolaires.

97 LMS - Local Management of Schools - veut dire que même les écoles qui choississent de rester sous l'égide des LEA sont devenues totalement responsables de leurs propres 
budgets :- état des lieux, fournitures scolaires, recrutement et rémunération des professeurs. établissement a opté de quitter définitivement la tutelle de la LEA en faveur d'un financement directe du Ministère (depuis l'année dernière appelé Department for Education ou DFE) en échange de quelques garanties, bien sûr.

Etant donné que nous avons un système pour l'Angleterre et le Pays de Galles (que je décris actuellement), un autre pour l'Ecosse, sans parler de l'Irlande du Nord ou des différences de statut organisationnel (LMS $=$ LEA $/$ GMS $=$ Ministère) vous allez comprendre que tout ceci reste très fragmenté du point de vue de l'organisation pratique de l'infrastructure mais avec une seule grande différence. Maintenant il y a un curriculum national pour tous les jeunes de 5 à 16 ans. Ce programme national définit le contenu général et les objectifs par étapes mais n'essaie pas de définir la méthodologie ni le détail. Cela n'a jamais existé auparavant. Le National Curriculum Framework (ou cadre général) s'adresse aux matières et aux thèmes, et aussi aux activités voisines comme, par exemple, des « projets ».

Les matières de base 'FOUNDATION SUBJECTS' sont

- CORE (noyau)

- anglais

- mathématiques

- sciences

- gallois (dans les écoles galloises du Pays de Galles).

\section{OTHERS (autres)}

- Technologie

- Géographie

- histoire

- une langue étrangère

- dessin

- musique

- éducation physique

- gallois (dans les écoles anglaises du Pays de Galles).

En plus, chaque établissement doit assurer l'éducation religieuse (religions comparées).

103 Je crois que du point de vue du géographe professionnel, travaillant dans l'éducation, l'élément le plus intéressant est l'importance des thèmes transversaux :

- la compréhension économique et industrielle

- la transition école - travail

- l'éducation pour l'environnement

- l'éducation pour la santé

- la citoyenneté (individuelle, familiale, communale, nationale, européenne, et internationale, y compris les dimensions légales et politiques).

C'est justement cette insistance sur l'importance de ces thèmes transversaux dans le National Curriculum, lié au fait que la géographie est reconnue comme matière de base destinée à tout le monde, qui présente le plus grand intérêt pour l'avenir. Nous espérons que nous allons quitter définitivement la notion de «matière » en faveur de de la notion de «mortier » en ce qui concerne la position de la géographie dans cette construction nouvelle. 
105 J'ajouterai aussi un autre thème, peut-être le plus «transversal» de tous (et pour lequel j'ai une responsabilité nationale au Royaume Uni, ce qui explique ma présence ici parmi vous) : la dimension européenne dans l'éducation où la géographie joue un rôle clef. Nous avons déjà entendu que la géographie était utile pour faire la guerre. C'est indispensable pour faire la paix!

106 Pour terminer je voudrais revenir à la position de l'enseignement de la géographie. Au début dans le "guideline »s du National Curriculum tous les élèves allaient devoir étudier les « foundation subjects » jusqu'à l'âge de 16 ans. A la suite des réclamations de beaucoup d'organisations professionnelles, de professeurs et de parents, on a jugé prudent «d'alléger» les progammes obligatoires entre 14 et 16 ans. Maintenant un choix peut se faire à 14 ans entre la géographie et l'histoire, le dessin et la musique. Ceci à l'issue d'une «Consultation » très élargie qui a eu lieu en 1991. Ceci a provoqué beaucoup de débats et les camps restent toujours divisés.

107 A Key Stage 4 (14 -16 ans) notre Ministre (qui chez nous s'appelle Secrétaire d'Etat) a accepté que les élèves qui suivent les cours de géographie comme matière principale, ne soient plus obligés de suivre un cours d'histoire, matière principale, et vice-versa. Autre possibilité, un élève peut désormais suivre deux cours subsidiaires de géographie + histoire. Donc pour vous, chers géographes/historiens, il existe plusieurs permutations :

1. histoire ou géographie principale (une matière)

2. histoire principale plus géographie principale (deux matières)

3. principale plus subsidiaire (une matière et demie)

4. subsidiaire plus subsidiaire (une matière).

La réalité voudra sans doute que peu d'écoles seront en mesure d'offrir toute la gamme et un choix « pernicieux » (je cite les professeurs concernés) va s'opérer. Pour « organiser » le National Curriculum, 4 «Etapes Clefs » (Key Stages) de 1 à 4, ont été identifiées. Pour chacun des «foundation subjects " (matières de base) il y a un groupe de spécialistes composé de représentants de beaucoup de secteurs, y compris les employeurs.

Il y a aussi des « attainment targets» (cibles ou buts de performance) pour chaque Etape Clef. Donc, à la fin de chacune de ces étapes il y aura une évaluation nationale de progrès par les SATs (standard assessment tasks) un contrôle à 7 ans et un autre à 11 ans en anglais, maths, et sciences, un contrôle à 14 ans pour toutes les matières.

110 Les TATs (teacher assessment tasks) sont des évaluations élaborées par les professeurs pour les autres matières à partir de 7 ans qui ne sont pas évaluées par les SATs. Tout le système sera à la charge des professeurs eux-mêmes, administrativement parlant...

111 Il faut noter - vous le savez, j'en suis persuadé, aussi bien que moi, - que la question des niveaux et surtout le testing ont provoqué une telle tempête que tous les syndicats menacent de faire grève et paralysie générale. L'été promet cette année !

112 Merci de m'avoir invité et de m'avoir écouté. 
L'instauration d'une pédagogie progressive dans l'enseignement de la géographie générale (I) : du concret à l'abstrait

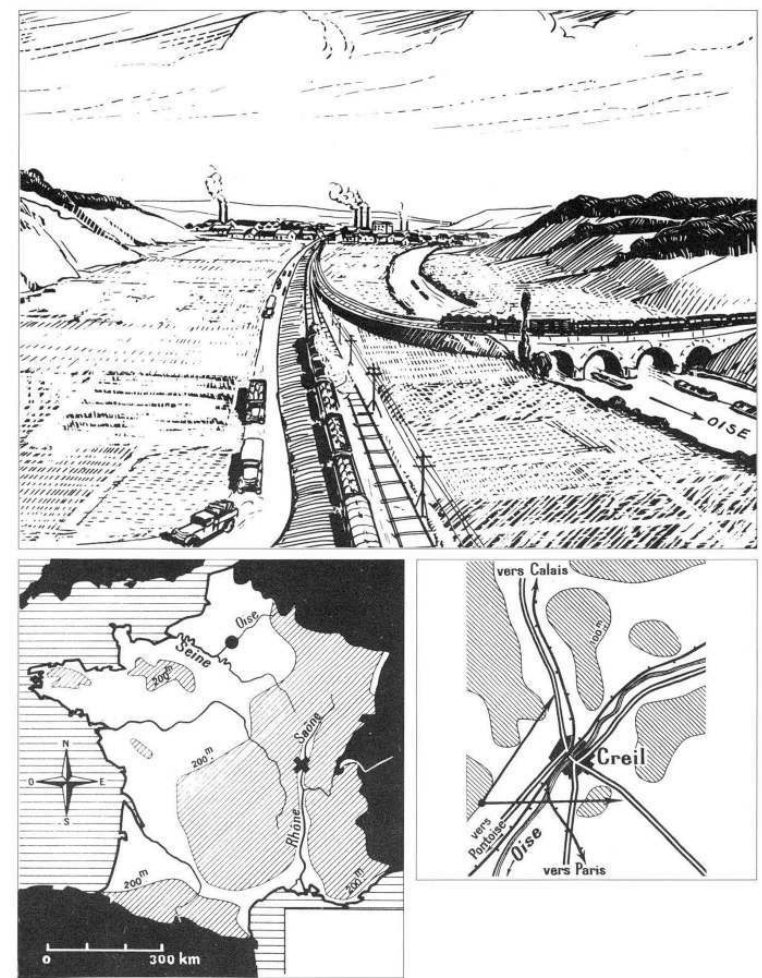

La vallée de l'Oise à Creil (Oise) : • = Lieu représenté par la 1ère gravure, ; $x=$ Lieu représenté par la 2ème gravure

A. Merlier, G. Leroux : Géographie (Paris, A. Hatier, 1951) p. 18 
L'instauration d'une pédagogie progressive dans l'enseignement de la géographie générale (II) : du proche au lointain, à travers les plans et les cartes

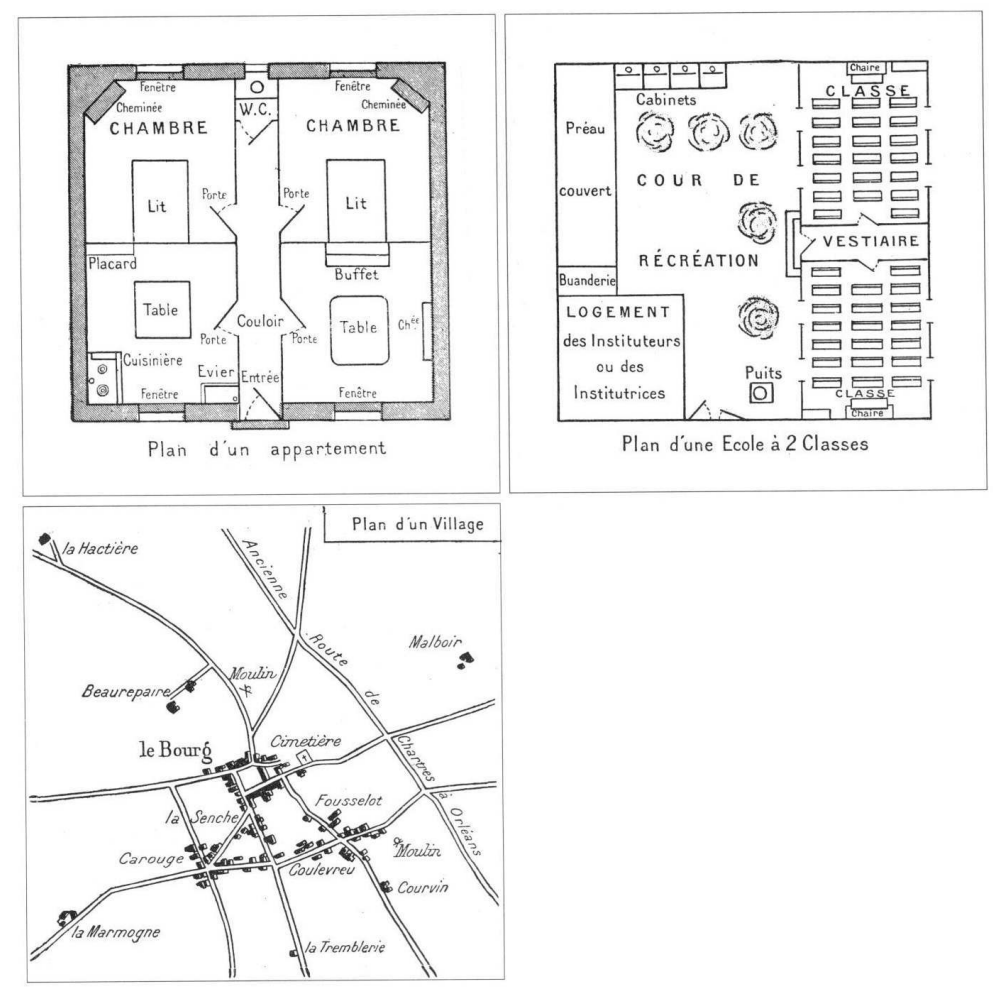

E. Driault, M. Randoux, M. Bizeau : Cours complet de géographie (Paris, Larousse, 1925)p. 2.

\section{Clara Dematteis}

Quels sont les objectifs en géographie régionale?

114 J'ai beaucoup appris ce matin, mais trouve toujours difficile d'enseigner la géographie régionale, après de nombreuses années d'enseignement. Chacun de nous voudrait " changer de plan » mais nous sommes liés aux programmes. Et c'est toujours un choix, si l'on veut couper au péril de faire une description-catalogue de phénomènes et de choses. Nous avons suivi ce matin des exemples de leçons qui ont fait des choix, d'arguments et de finalités.

Je voudrais parler de finalités et objectifs de savoir-faire et même savoir-être, à travers la dimension civique de la géographie dont parlait ce matin le Recteur Blanchet.

116 Je prendrai un exemple autre que celui du Languedoc-Roussillon, lié au Piémont italien. En Italie la géographie des régions commence dans l'enseignement primaire à peu près à l'âge des enfants que nous avons vus ce matin, avec une présentation de leur région. Dire le Piémont c'est parler de Turin, pôle central (peut-être aussi une périphérie ?) et Turin c'est l'automobile, la FIAT ; mais le Piémont est aussi la montagne, toute proche pour le ski, la promenade, liée très intimement à FIAT : l'énergie pour l'industrie en vient, les idées d'Europe, le monde des travailleurs pour les usines, et même Agnelli. C'est un choix de parler des lieux, d'abord. 
117 Il y a là des objectifs de connaissances, de savoir-faire (mettre les lieux sur la carte), mais aussi des objectifs éducatifs, prendre conscience des différences territoriales dans le Piémont: montagne mais plaine aussi où passent l'autoroute, les routes du Nord de l'Italie. C'est faire comprendre aux enfants que le territoire est à la fois lieu de ski, de divertissement en périphérie de la ville, mais que la montagne a aussi son importance, ses traditions, ses valeurs. Il y a là un objectif éducatif lié à l'écologie, l'environnement. Plus tard on parle du Piémont dans nos écoles secondaires, le premier cycle, aux élèves de 12-13 ans.

118 Je parlerai du Piémont selon une approche historique, non pas de Turin en tant que capitale, mais à travers ses liens avec le sud de l'Italie d'où sont arrivés beaucoup de parents d'élèves (1950-1970) pour travailler dans les usines. En liaison aussi avec le démarrage économique du Piémont, les élèves retrouveront leurs racines. Turin est lié au sud. L'objectif sera les connaissances approfondies, le savoir-faire et le savoir-être en connaissant ses origines, les liens avec l'industrie. Il y a là une forme d'acquisition du respect pour l'autre, dans sa diversité. Y compris face à des émigrés venus d'encore plus loin.

119 La géographie du Piémont est abordée aussi en second cycle, dans les filières économiques. Dans cette troisième étape les liaisons entre Turin et le Piémont se situent hors du pays car l'industrie automobile est aussi liée à l'Europe où de nombreuses usines automobiles ont été fermées. L'industrie est en crise, et cela va au-delà de l'Italie, ce qui est l'occasion d'élargir le panorama de l'étudiant. L'objectif de formation passe par l'ouverture au monde, en voyant les liens entre cette petite partie du monde et le reste de la planète, qui a ses propres choix. La géographie régionale ainsi choisie doit prendre des objectifs éducatifs de façon transversale par rapport aux autres matières. Ce que l'on ne manque pas de faire. 
A. Merlier, G. Leroux : Géographie (Paris, A. Hatier; 1951) p. 11.

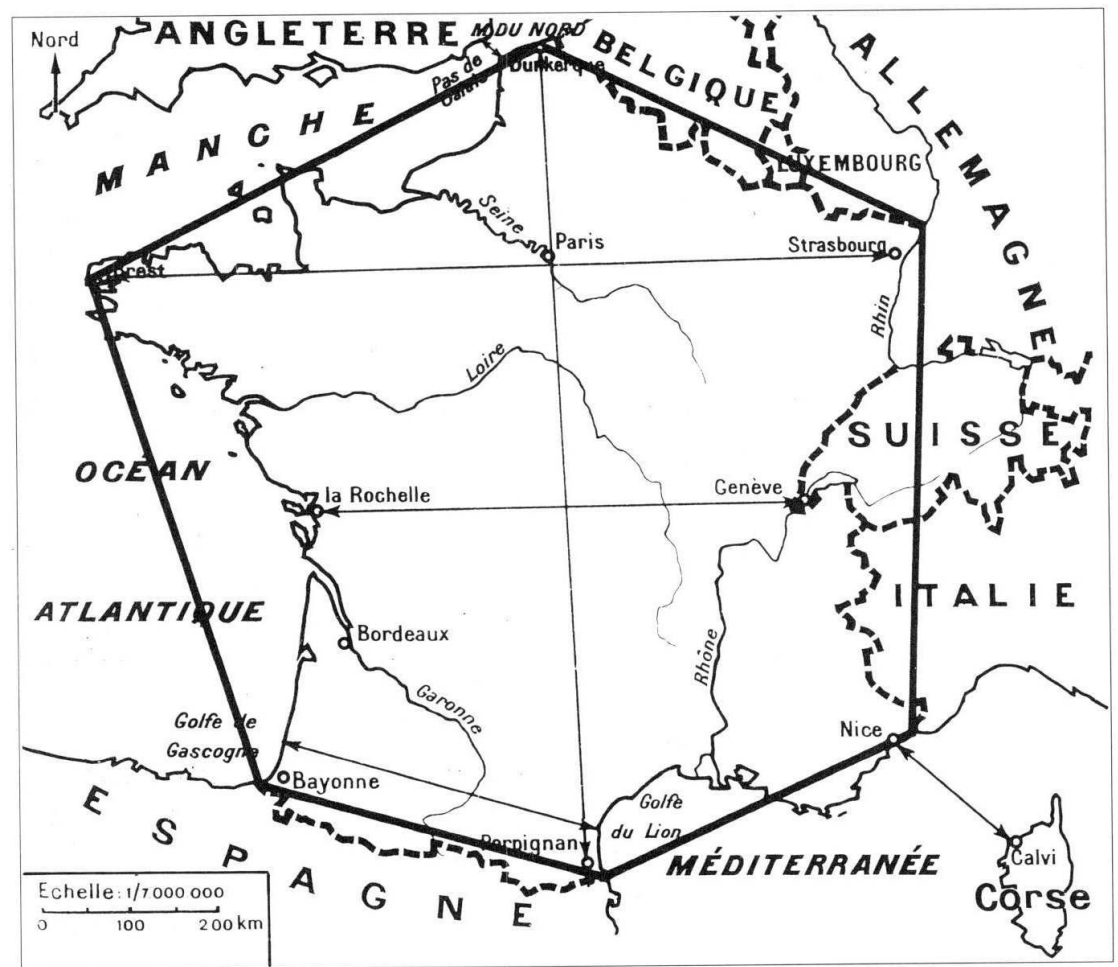

Géographie nationale (I)Le point de vue esthétique : la France est un pays harmonieux.

\section{AUTEURS}

\section{ROBERT FERRAS}

Professeur des universités, directeur de l'IUFM de Montpellier

\section{DOMINIQUE BORNE}

Doyen de l'Inspection Générale, Président du jury du CAPES d'histoire géographie

\section{PIERRE DESPLANQUES}

Inspecteur Général de l’Education Nationale

\section{TONY MALE}

Directeur du Central Bureau for Educational Visits \& Exchanges

\section{CLARA DEMATTEIS}

Chargée de cours à l'université de Turin, spécialiste de didactique de géographie au sein de

l'Association des Professeurs d'histoire et géographie d'Italie 